\title{
Paroxysmal head pain with backward radiation: will epicrania fugax go in the opposite direction?
}

\author{
María Luz Cuadrado • Lidia Gómez-Vicente • \\ Jesús Porta-Etessam • María Azahara Marcos-de-Vega • \\ Beatriz Parejo-Carbonell · Jorge Matías-Guiu
}

Received: 25 October 2009/Accepted: 9 November 2009/Published online: 21 November 2009

(C) Springer-Verlag 2009

\begin{abstract}
Epicrania fugax (EF) has been recently described as a paroxysmal head pain starting in a focal cranial area of the posterior scalp and rapidly spreading forward to the ipsilateral eye or nose along a linear or zigzag trajectory. Here we report two patients presenting with the same clinical features, except for the starting site and the direction of the pain. Unilateral pain paroxysms occurred on either side of the head, with a quick backward radiation along a linear trajectory. The pain always stemmed from a particular point located at the fronto-parietal region, and reached the parieto-occipital region in several seconds. The symptoms did not fit any of the acknowledged headaches and neuralgias, and might correspond to a reverse variant of EF.
\end{abstract}

Keywords Epicrania fugax - Cranial neuralgia ·

Supraorbital neuralgia Trigeminal neuralgia .

Primary stabbing headache

\section{Introduction}

Epicrania fugax $(\mathrm{EF})$ is a novel syndrome recently identified by Pareja et al. [1] in ten patients with a stereotypical type of head pain. Their clinical picture was essentially characterized by unilateral pain paroxysms starting in a particular focal area of the posterior scalp, and rapidly spreading forward along a linear or zigzag trajectory to

M. L. Cuadrado ( $\varangle)$ · L. Gómez-Vicente · J. Porta-Etessam · M. A. Marcos-de-Vega · B. Parejo-Carbonell · J. Matías-Guiu Department of Neurology, Hospital Clínico San Carlos and Universidad Complutense, Profesor Martín Lagos s/n, 28040 Madrid, Spain

e-mail: mlcuadrado@med.ucm.es reach the ipsilateral eye or nose in one to a few seconds (Fig. 1a). The attacks could be either spontaneous or triggered by touch on the stemming point, and took an electric or stabbing character. Between the paroxysms some patients stayed asymptomatic, while others felt slight pain or tenderness at the stemming zone. Since the first description of EF, a few more cases have been reported $[2,3]$.

Here we report two patients presenting with unilateral shooting pain paroxysms starting in an anterior cranial point and radiating backwards, whose features were otherwise similar to those of EF. Their symptoms did not fit any of the acknowledged headaches and neuralgias, and might correspond to a reverse variant of EF.

\section{Case reports}

\section{Patient 1}

A 35-year-old man started having paroxysmal attacks of head pain which could not be related to any event. He denied prior headaches, trauma, or important diseases, except for a past herpetic meningoencephalitis with good response to treatment and no sequels. Within each attack the pain was strictly unilateral, more often right than left. It always stemmed from a particular point at the frontoparietal region, and then moved backwards along a linear arc to the parieto-occipital region. It took $40-50 \mathrm{~s}$ to reach the end. The quality of this moving pain was described as electric, and its intensity was moderate (around 5 in a 10-point rating scale). There were no autonomic symptoms or other accompaniments. At onset he experienced 2-3 attacks every day, but pain frequency decreased gradually to $1-2$ attacks per week through the 
Fig. 1 a Trajectory of pain paroxysms in the original series of epicrania fugax (modified from Pareja et al. [1]) . b Trajectory of pain paroxysms in our two patients
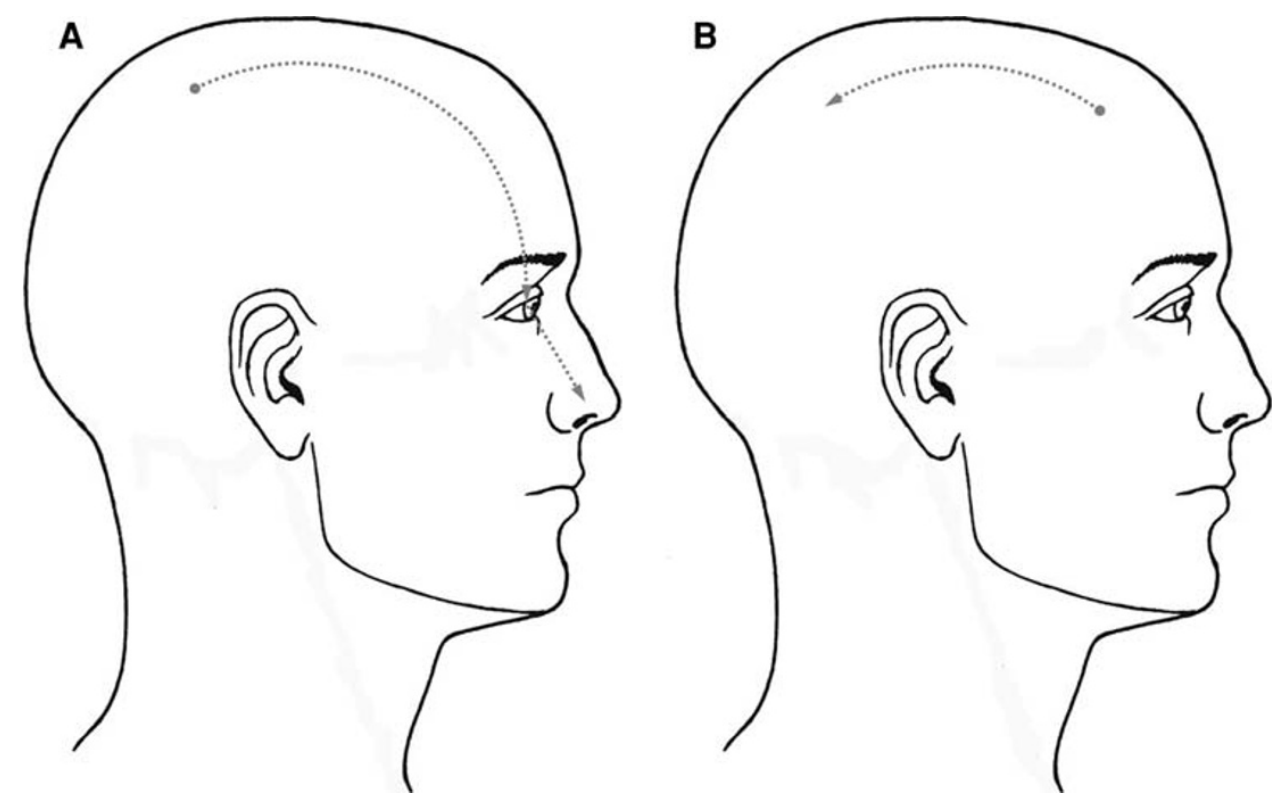

next 4 months. There were no apparent triggers. Between the paroxysms the patient did not feel any pain or other sensory symptoms in the scalp.

Both the general and the neurologic examinations were normal. Palpation of the supraorbital, auriculotemporal, minor occipital and greater occipital nerves did not evoke any abnormal sensation. Neither skin changes nor signs of sensory dysfunction could be detected at the edges or along the path of the pain. Routine blood work-up, including erythrocyte sedimentation rate, thyroid tests and immunological screening, and magnetic resonance imaging (MRI) of the brain did not show any abnormalities.

\section{Patient 2}

A 26-year-old woman with no relevant medical history and no prior history of headaches started presenting a paroxysmal type of head pain without any precipitating event. The pain always started in a fronto-parietal point, and rapidly moved backwards following a linear trajectory, reaching the parieto-occipital region in 5-6 s. It could occur on either side of the head, but was usually rightsided. Pain quality was described as electric, and pain intensity was moderate (around 6 in a 10-point rating scale). There were no associated symptoms. Otherwise, she had not noticed any interictal tenderness or triggers. The episodes recurred with a frequency of 2-3 per day for 2 weeks, but she did not take any medication during that time. Then the pain paroxysms remitted spontaneously, and there were no relapses during a follow-up period of 3 months.

Physical and neurological exams, including inspection and palpation of the painful areas and palpation of pericranial nerves, were normal. All ancillary studies, including routine blood tests with erythrocyte sedimentation rate, thyroid tests, immunological screening and MRI of the brain, were also normal.

\section{Discussion}

Here we present two patients with a non-characterized never before described type of head pain, with almost identical features. There were some differences regarding the temporal features (attack duration, attack frequency and long-term course), but their main characteristics were roughly the same. Unilateral pain paroxysms occurred on either side of the head, with a quick backward radiation along a linear trajectory. The pain always stemmed from a particular point located at the fronto-parietal region, and reached the parieto-occipital region in several seconds (Fig. 1b). The pain was described as moderate and electric, and recurred at intervals of hours or days with no evident triggers. There were no autonomic symptoms or other pain accompaniments. Between the paroxysms, both patients remained asymptomatic. In neither case could the pain be attributed to an underlying disorder.

Sudden occurrence of short cephalic pain paroxysms is the core feature of primary stabbing headache (PSH). However, PSH is typically characterized by motionless localized ultrashort stabs, which start and end in the same point [4-6]. This point commonly changes among episodes, though some patients tend to have all their stabs within the same zone. The stabs are normally single, and only very rarely appear in volleys $[6,7]$. Consecutive stabs usually happen in the same point, but may sporadically 
shift from one region to another. Theoretically, a series of separate stabs may give an illusion of movement, but a continuous trail as the one depicted by our patients has never been described. Despite some similarities with PSH, our patients seem to have a different headache disorder.

The existence of a focal stemming area, the electric character and the narrow pattern of radiation are features consistent with a neuralgic or neuralgiform syndrome. However, our patients' symptoms were not typical for any of the acknowledged cranial neuralgias. The possibility of a supraorbital neuralgia might be considered, since their pain was felt in the area supplied by the supraorbital nerve. Nonetheless, supraorbital neuralgia produces a normally continuous, forehead pain with tenderness in the supraorbital notch $[4,8,9]$. First division (V-1) trigeminal neuralgia might be also borne in mind, since the paroxysms of pain occurred in the territory of V-1. However, the paroxysms of $\mathrm{V}-1$ trigeminal neuralgia are mostly located in the orbit and forehead, tend to spread to V-2 and V-3 areas, and can be triggered from $\mathrm{V}-1, \mathrm{~V}-2$ and $\mathrm{V}-3$ territories and by multiple precipitating mechanisms $[4,10,11]$. Besides, both pain intensity and suffering are much greater in patients with trigeminal neuralgia than in these two patients. Indeed, neither of them required drug therapy. SUNCT also produces neuralgiform attacks in the territory of V-1, but the differences with SUNCT were even more evident. SUNCT attacks are characterized by orbital or periorbital pain together with prominent lacrimation and conjunctival injection. Moreover, the attacks are usually triggered by mechanical stimuli acting on trigeminal or extra-trigeminal territories [4, 12-14].

EF has been recently described as a paroxysmal head pain starting in a particular focal area of the posterior scalp, and rapidly spreading forward to the ipsilateral forehead, eye or nose along a linear or zigzag trajectory (Fig. 1a). Most attacks are spontaneous, although some patients can also have attacks triggered by touch stimuli on the stemming point. Pain intensity is usually moderate or severe, and pain quality is stabbing or electric. The pain may be followed by ocular or nasal autonomic signs at the end of the attacks, but many patients lack pain accompaniments. The complete sequence is very brief, lasting just one or a few seconds. Between the paroxysms a slight painful or dysaesthesic sensation may remain in the stemming point, but many patients stay with no interictal symptoms at all. This syndrome awaits definite validation, but several cases have already been reported [1-3]. Our patients showed an identical clinical picture, except for the starting site and the direction of the pain. Instead of stemming posteriorly and radiating forwards, the pain started in an anterior cranial region and then moved backwards (Fig. 1b). Otherwise, the paroxysms displayed the same spatial, temporal and quality features as those described for EF. The episodes of Patient
1 were longer than those of Patient 2 or those described for EF up to now, but still fell within a range of seconds. Accordingly, the pain of these two patients might be considered a reverse variant of EF. Even so, further similar cases should be searched. Alvaro et al. [2] has already included one patient with posterior radiation in their series of EF, but a complete clinical description of this case has not been published yet.

The pathogenesis of EF is largely unknown [1]. The origin of the pain is possibly peripheral, given the presence of a fixed stemming area that may have local sensory dysfunction and may trigger the paroxysms in some patients. The subsequent radiation might be caused either by peripheral extension from the point of origin to adjacent nerve fibres or nerve branches, or to central extension through the trigeminal nucleus caudalis. Interestingly, in both former EF cases and these cases, the pain had similar boundaries, either starting or ending at the limits between trigeminal and occipital-or C-2-territories. If the present head pain is to be considered $\mathrm{EF}$, the direction of the pain would change among patients. We cannot be certain whether pain propagation-and pain direction-in each type is mediated by peripheral or central mechanisms. The duration of attacks in Patient no. 1 was longer than the normal duration in paroxysmal neuralgias [11], and this may reflect an important role of central processing.

In conclusion, we have encountered two patients with a distinctive type of head pain which resembled EF but had an opposite radiation. Their features did not fit well other acknowledged headaches or neuralgias, and might correspond to a reverse variant of EF. Further observations are required for a definite characterization of this headache condition.

Conflict of interest None.

\section{References}

1. Pareja JA, Cuadrado ML, Fernández-De-Las-Peñas C, Caminero $\mathrm{AB}$, Nieto C, Sánchez C et al (2008) Epicrania fugax: an ultrabrief paroxysmal epicranial pain. Cephalalgia 28:257-263

2. Alvaro LC, García JM, Sádaba F (2008) Epicrania fugax: tipos clínicos en cuatro nuevos casos. Neurología 23:732-733 (abstract)

3. Guerrero AL, Porta-Etessam J, Cuadrado ML, García-Ramos R, Herrero S, Lapeña T et al (2009) Epicrania fugax: six new cases and therapeutic results. J Neurol 256(Suppl 2):S42-S43 (abstract)

4. Headache Classification Subcomittee of the International Headache Society (2004) The international classification of headache disorders, 2nd edn. Cephalalgia 24(Suppl 1):9-160

5. Pareja JA, Ruiz J, De Isla C, Al-Sabbah H, Espejo J (1996) Idiopathic stabbing headache (jabs and jolts syndrome). Cephalalgia 16:93-96

6. Sjaastad O, Pettersen H, Bakketeig LS (2002) The Våga study of headache epidemiology II. Jabs: clinical manifestations. Acta Neurol Scand 105:25-31 
7. Sjaastad O, Pettersen H, Bakketeig LS (2005) Long-lasting cephalic jabs (?). The Våga study of headache epidemiology. Cephalalgia 25:581-592

8. Sjaastad O, Petersen HC, Bakketeig LS (2004) Supraorbital neuralgia. Vågå study of headache epidemiology. Cephalalgia 25:296-304

9. Pareja JA, Caminero AB (2006) Supraorbital neuralgia. Curr Pain Headache Rep 10:302-305

10. Sjaastad O, Pareja JA, Zukerman E, Jansen J, Kruszewski P (1997) Trigeminal neuralgia. Clinical manifestations of first division involvement. Headache 37:346-357

11. Pareja JA, Cuadrado ML, Caminero AB, Barriga FJ, Barón M, Sánchez del Río M (2005) Duration of attacks of first division trigeminal neuralgia. Cephalalgia 25:305-308
12. Pareja JA, Shen JM, Kruszewski P, Caballero V, Pamo M, Sjaastad O (1996) SUNCT syndrome. Duration, frequency and temporal distribution of attacks. Headache 36:161-165

13. Pareja JA, Cuadrado ML (2005) SUNCT syndrome. An update. Expert Opin Pharmacother 6:591-599

14. Cohen AS, Matharu MS, Goadsby P (2006) Shortlasting, unilateral, neuralgiform headache attacks with conjunctival injection and tearing (SUNCT) or cranial autonomic features (SUNA). A prospective clinical study of SUNCT and SUNA. Brain 129:2746-2760 